\title{
Health-related quality of life of among elders in rural China: the effect of widowhood
}

\author{
Jianfang Zhou $^{1}$ (D) Norman Hearst $^{2}$
}

Accepted: 7 June 2016/Published online: 13 June 2016

(c) The Author(s) 2016. This article is published with open access at Springerlink.com

\begin{abstract}
Purpose China has an enormous and rapidly growing population of widowed elders. Little is known about how losing a spouse affects elders' health-related quality of life (QOL), especially in the rural areas where most Chinese elders live. This article analyzes QOL data collected in 2014 among rural Chinese elders to address this question. Methods SF12 questionnaires and information about individual and household characteristics were collected from 3053 elders aged 60 and above in rural China. We compared the physical component summary (PCS) and mental component summary (MCS) scores between 1925 married elders and 1060 widowed elders in a bivariate model stratifying by gender and age group and in a general factorial ANOVA multivariate analysis that examined and controlled for other predictors of PCS and MCS scores including education, chronic disease, and family and household factors.

Results Widowed male and female elders' physical health and mental health were in decline with age. Widowed men had lower PCS and MCS scores than married men. Widowed women also had lower PCS scores, but differences in MCS scores did not reach statistical significance. In
\end{abstract}

Jianfang Zhou

jianfangzhou@hotmail.com

Norman Hearst

hearstn@fcm.ucsf.edu

1 Institute of Humanities and Social Sciences, Nanjing University of Posts and Telecommunications, Wenyuan Road 9, New Yadong District, Nanjing 210023, China

2 Departments of Family and Community Medicine and of Epidemiology and Biostatistics, University of California, San Francisco, CA 94143, USA multivariate analysis, widowhood was associated with lower PCS and MCS scores overall. Support from children was associated with better QOL and, based on interaction analysis, appeared to mitigate negative effects of widowhood.

Conclusions Widowed rural elders in China have lower physical and mental quality of life than their married counterparts. These elders rely on their children for care, and a supportive family is associated with better QOL.

Keywords Widowhood $\cdot$ QOL $\cdot$ Elders · Rural China

\section{Background}

Data from the Sixth Chinese National Population Census in 2010 show that there were 47.7 million widowed elders in China, accounting for $26.9 \%$ of the total population over age 60 , and that this rate was slightly higher $(28.8 \%)$ in rural areas. The number of widowed elders is expected to grow rapidly in China, reaching 118.4 million by 2050 [1]. The rapid increase in the number of elderly coupled with very low fertility rates in recent decades means that the number of elderly with few or no offspring to care for them will inevitably increase dramatically [2]. Therefore, the spouse will play a greater role in late life care. We conducted this study to examine the impact of widowhood on quality of life among elders in rural China.

Spouses play a protective role that might be expected to improve elders' health-related quality of life (QOL) in ways ranging from daily care and accident prevention to spiritual consolation [3, 4]. QOL is a complex concept. The WHO QOL Group defined QOL as individuals' perceptions of their position in life in the context of the culture and value systems in which they live and in relation to their 
goals, expectations, standards, and concerns [5]. Bowling and Gabriel postulate that QOL is a multi-dimensional collection of objective and subjective areas of life, the parts of which can affect each other as well as the sum [6]. An elder who loses a spouse may not only lose a certain degree of economic or social support. Widowhood may also bring loneliness and considerable difficulty in daily life. Assessing QOL of widowed elders and analyzing how losing a spouse affects elders' QOL are thus important for designing effective health promotion strategies for widowed elders in China.

Research on QOL in China began fairly recently. A few studies of QOL among Chinese elders have been published in the past few years, though none has focused on the role of widowhood or been conducted in rural areas. These studies found that sex, age, and household economics were related to QOL [7-10]. Luo and Chen's studies did not find marital status related to elders' QOL [7, 8], but Feng and Tian's studies showed that scores of both the physical and mental health components of quality of life of married elders were higher than unmarried elders (including single, divorced and widowed) $[9,10]$. One study in urban China showed that more care from children was associated with better QOL [11]. Studies examining the relationship of living status and QOL among elders have given conflicting results. Cheng and Li's study found that elders living with children had better overall QOL [12-14]. But Wei found that elders living only with their spouse scored better than those living with children [15].

Although no previous study has focused on Chinese widowed elders' QOL, there are studies indicating increased standardized mortality among widowed elders, especially those newly widowed [16-20]. Numerous international studies have shown that married people have lower mortality than the unmarried [21-23]. Studies in England, Australia, and Norway [24-28] also found apparent effects of widowhood on physical and mental health and morale. But other studies [29, 30] found no health advantages for older women living with a spouse compared with those living alone. Some researchers suggest that reactions to bereavement are strongly influenced by culture and ethnicity [31, 32]. Different stages of bereavement also may have different effects on well-being, which in general tends to bounce back to its previous level following major adverse life events. How widowhood affects QOL of Chinese elders remains to be quantified, especially in rural areas. Better understanding this may help to explain subsequent morbidity and mortality and suggest ways to promote the health of these elders. This article analyzes QOL data collected in 2014 among rural Chinese elders to address these questions.

\section{Methods}

\section{Study sample}

The definition of rural elders in this study was a person age 60 and above who mainly lived in a rural area during the previous year. Since regional differences might influence QOL, this study sampled from three provinces, one each from the east (Jiangsu Province), the central (Henan province) and the western (Qinghai Province) regions of China. These provinces represent a range of social and economic rankings. One county was selected from each of the provinces by judgment sampling. The criteria were that in 2013 the county: (1) had more than 30,000 residents over age 60 ; (2) was mainly rural; and (3) had a social economy near the median level for the province. We selected two townships in each county by simple random sampling and obtained a list of 530 randomly selected families with elders using the local household registration information system in each township. We sampled 530 families to yield a total desired sample size of 3000 with a small margin of safety for non-respondents. We sampled two townships in each county because some rural townships have less than 1000 families with elders. For families with more than one elder, one was randomly selected. We successfully interviewed a total of 3053 elders. For the $4.0 \%$ of the sample not interviewed (the rate was similar in married and widowed elders), the main reason was not being at home during the approximately one-week period of interviews. Among the participants, there were 1925 married elders, 1060 widowed elders, and 68 single elders. The 68 single elders who were not widowed were excluded from the study.

\section{Data collection method}

This study was approved by the Nanjing University of Posts and Telecommunicates Ethics Committee. All participants gave informed consent prior to inclusion in the study. After conducting a pretest in one of the sample counties in January 2014, formal investigation took place from March to May. Local trained investigators interviewed elders (or their caregivers for the $8.3 \%$ of elders who could not answer questions by themselves due to their health status) at their home. In each county, we selected 30 interviewers from local community workers. The main criteria were: (1) age 30-50; (2) junior high school education or higher; (3) availability to work full time during the survey week; and (4) good patience, communication skills, and responsibility. 


\section{Variables}

\section{Individual characteristics}

Based on previous studies [7-9, 33-40], this study collected data on respondents' date of birth (this was converted into age in years and analyzed in age strata and as a continuous variable); sex; highest level of education (illiterate, elementary school, junior high school or higher); whether suffering from chronic diseases (yes vs. no); and current marital status (married vs. widowed). For those widowed, we asked the year in which the spouse had died; we did not ask the exact date because our pre-test indicated that widows often could not remember this.

\section{Household factors}

We constructed questions to assess household factors based on variables associated with QOL in previous research [34-39]. Participants were asked: whether they lived with at least one of their children (yes vs. no); frequency of children asking about their health (always, often, seldom, or never asked); whether children were one of their main source of income (yes vs. no); and whether children were one of their main caregivers when they were ill (yes vs. no).

QOL This study used SF-12 (V2 in Chinese) to measure the health-related QOL of elders. The scale was developed by the Health Research Institute of the New England Medical Center in Boston, USA. It comprehensively summarizes the content of physical, psychological, and social aspects of health [40]. The scale is composed of 12 items divided into eight dimensions: physical functioning $(\mathrm{PF})$, role physical (RP), bodily pain (BP), general health $(\mathrm{GH})$, vitality (VT), social functioning (SF), role emotional (RE), and mental health (MH). Scoring was based on the scale users' manual [40]. The range of each dimension score was from 0 to 100 with higher scores meaning higher QOL. From these scores, the physical component summary (PCS) and mental component summary (MCS) scores were also calculated based on the manual. A score higher than 50 represents above the original norm (USA) and higher scores mean higher QOL. Lam tested SF12 V2 (Chinese version) in the general Chinese population and confirmed it had good reliability and validity [41]. Xiao tested it on Chinese elders who lived in pension institutions or at home and also confirmed that it had good reliability and validity. [42]

\section{Analysis}

We used Epidata 3.2a to record responses and SPSS 17.0 for analysis. Interviewers and investigators double-checked all questionnaires in the field, so no questionnaire items were missing for more than $1 \%$ of respondents. In the few cases of missing data, we substituted the mode value (for qualitative variables) or mean value (for quantitative variables) in accordance with the SF12 users' instructions and for consistency with other studies. We compared the differences of the personal and household factors and PCS and MCS scores between widowed and married elders using the Chi-square test and one-way ANOVA. General factorial ANOVA analysis was then applied to examine the association of widowhood with PCS and MCS scores in a multivariate model to control for the effect of potential confounding variables. A second ANOVA model added interaction terms for widowhood with other significant predictors of QOL including age, sex, education, chronic disease, and family factors. The results of effects (measures of association) are shown as mean square, $F$ and $P$ value. Two-tailed $P<0.05$ is considered significant.

\section{Results}

\section{Individual and household characteristics}

As shown in Table 1, most of the widowed elderly participants were illiterate women with chronic diseases. Their average age was 73.5 years. Most of them lived with their children. Nearly $40 \%$ of them relied on their children as a main source of living expenses. Most reported that their children often asked about their health and cared for them when they were ill. Compared to married elders, widowed elders' personal characteristics showed significant differences: more were women, they were older, and more of them had chronic diseases. Judging from the individual characteristics, widowed elders had less personal health resources than the married elders. On the other hand, children provided more financial support and care to widowed elders than married elders. More of the widowed reported that their children were a main source of financial support, lived with them, and were a main caregiver when they were ill.

For married elders, $89.7 \%$ also reported that their partners were a main caregiver for them when they were ill. The percentage of children who were a main caregiver when ill was somewhat higher for female elders than male elders $(74.4 \%$ vs $67.9 \% ; P=0.010$ by Chi-square analysis; respondents could select more than one main caregiver when ill.)

Our sample included relatively few elders who were recently widowed, especially among the older age groups. Overall, only $5.7 \%$ had been widowed for less than a year and $71.9 \%$ had been widowed for over 5 years. The percentage widowed over 5 years reached a maximum of 
Table 1 Comparison of personal and family characteristics between widowed and married elders

\begin{tabular}{|c|c|c|c|}
\hline Variable & Married elders $(n=1925)$ & Widowed elders $(n=1060)$ & $P$ value \\
\hline Sex & $\begin{array}{l}\text { Male } 57.2 \%, \\
\text { Female } 42.8 \%\end{array}$ & $\begin{array}{l}\text { Male } 29.7 \% \text {, } \\
\text { Female } 70.3 \%\end{array}$ & $<0.001$ \\
\hline Age & $\begin{array}{l}\text { Average age } 67.51, \\
\text { Standard deviation } 6.01\end{array}$ & $\begin{array}{l}\text { Average age } 73.51, \\
\text { Standard deviation } 8.03\end{array}$ & $<0.001$ \\
\hline Education & $\begin{array}{l}\text { Illiterate } 50.8 \% \text {; } \\
\text { Elementary school } 34.8 \% \text {; } \\
\text { Junior high school or higher } 14.4 \%\end{array}$ & $\begin{array}{l}\text { Illiterate } 71.0 \% \text {; } \\
\text { Elementary school } 22.2 \% \text {; } \\
\text { Junior high school or Higher } 6.8 \%\end{array}$ & $<0.001$ \\
\hline Having chronic diseases & $\begin{array}{l}\text { Yes } 82.4 \% \\
\text { No } 17.6 \%\end{array}$ & $\begin{array}{l}\text { Yes } 85.8 \% \\
\text { No } 14.2 \%\end{array}$ & $<0.001$ \\
\hline Living with children & $\begin{array}{l}\text { Yes } 37.7 \% \\
\text { No } 62.3 \%\end{array}$ & $\begin{array}{l}\text { Yes } 52.8 \% \\
\text { No } 47.2 \%\end{array}$ & $<0.001$ \\
\hline Children as a main source of financial support & $\begin{array}{l}\text { Yes } 21.4 \% \\
\text { No } 78.6 \%\end{array}$ & $\begin{array}{l}\text { Yes } 41.9 \% \\
\text { No } 58.1 \%\end{array}$ & $<0.001$ \\
\hline Frequency of children asking about their health & $\begin{array}{l}\text { Frequently } 70.6 \% ; \\
\text { Once in a while } 24.2 \% \text {; } \\
\text { Almost never } 5.1 \%\end{array}$ & $\begin{array}{l}\text { Frequently } 71.0 \% \\
\text { Once in a while } 23.9 \% \\
\text { Almost never } 5.4 \%\end{array}$ & 0.577 \\
\hline Children as a main caregiver when ill & $\begin{array}{l}\text { Yes } 70.7 \% \\
\text { No } 29.3 \%\end{array}$ & $\begin{array}{l}\text { Yes } 93.2 \% \text {; } \\
\text { No } 6.8 \%\end{array}$ & $<0.001$ \\
\hline
\end{tabular}

$81.0 \%$ among widowers age 80 or higher. The small numbers recently widowed in many age/sex strata and the collinearity of length of widowhood with age precluded separate analysis of the effect of time since death of a spouse on QOL.

\section{QOL comparison between widowed elders and married elders}

Since age and gender are strong predictors of QOL and because previous studies have suggested widowhood may have different impact on QOL by sex and age [16-24, 28], we first compared QOL of married and widowed elders stratifying by gender and age group.

\section{Males}

Widowed male elders' physical health and mental health were in decline with age, but the decrease in physical health was more apparent (Fig. 1). One-way ANOVA analysis showed that compared with the male married elders, widowed men had lower PCS and MCS scores in general after controlling for age group $(P<0.01)$. When looking within specific age groups, we found that the PCS scores of widowed elders were significantly lower in the $60-65$ and $\geq 80$ years age groups. The MCS scores of widowed elders were significantly lower in 60-65, 65-70 and 70-75 years age groups.

\section{Females}

QOL of both widowed and married female elders also declined significantly with age. Their level of PCS dropped faster than MCS especially after age 70 (Fig. 2). In contrast to male elders, differences in MCS scores between widowed and married women did not reach statistical significance, either overall or within any age group. PCS was lower overall $(P<0.01)$ and in the $60-65$ age group.

\section{General factorial ANOVA analysis of the association of widowhood with PCS and MCS}

The comparison above shows that widowed elders' physical and mental health status tend to be poorer than married elders of the same sex and age groups, except for MCS among women. We used general factorial ANOVA analysis to examine the extent to which these differences might be due to confounding by other individual and family factors (Table 2).

Personal characteristics such as sex, age, marital status, education, and whether having chronic diseases were all significantly related to PCS. Elders who were female, older, widowed, illiterate, and with chronic diseases all scored lower. If their children asked more often about their health and were not a main source of financial support, they scored higher.

For MCS, elders who were older, widowed, illiterate, and with chronic diseases scored lower. If their children 


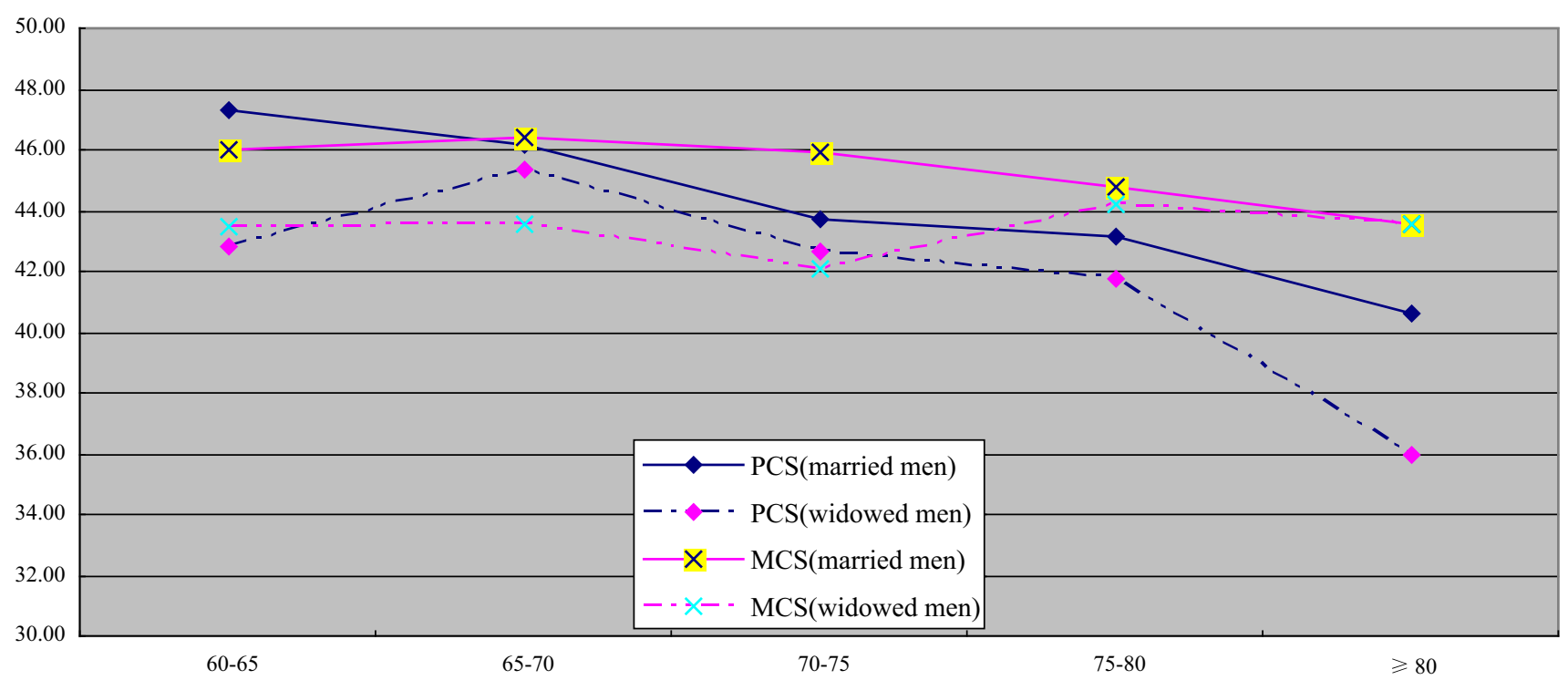

Fig. 1 QOL comparison between widowed and married men by 5-year age group

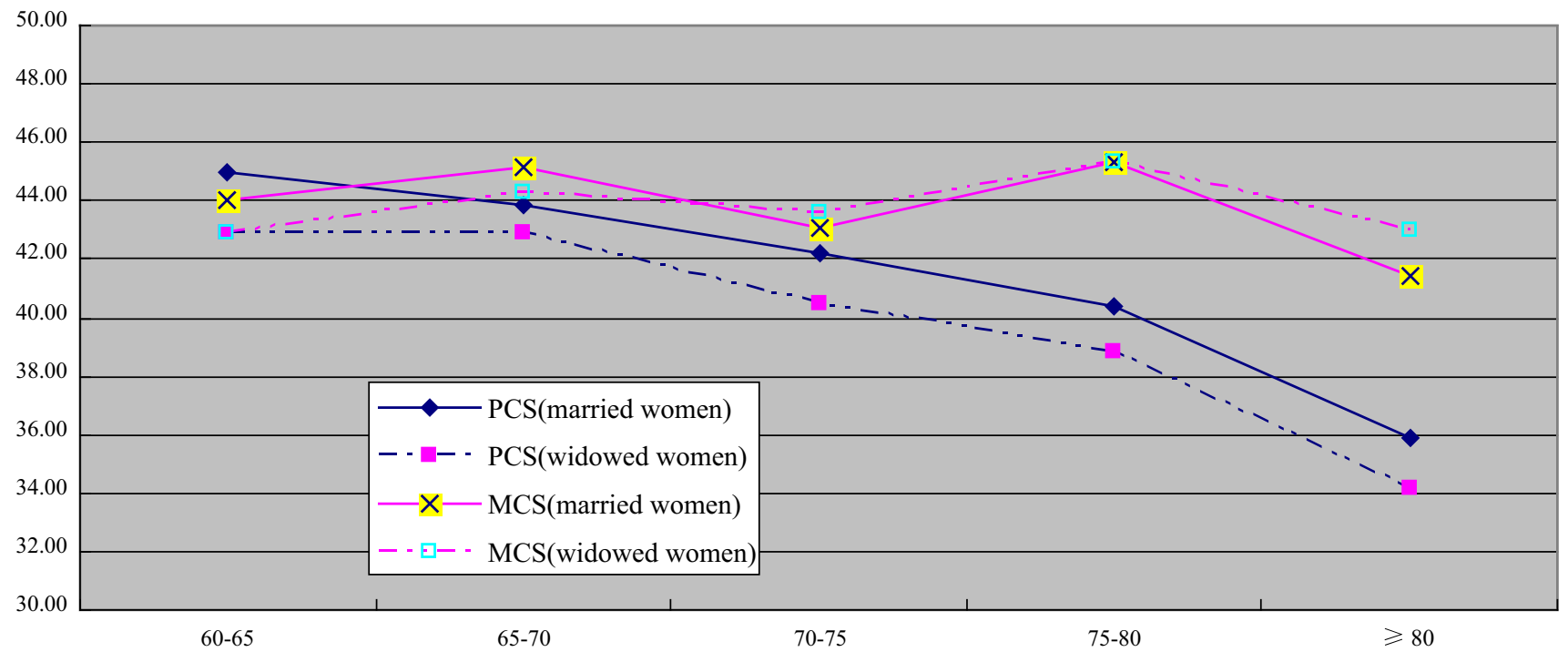

Fig. 2 QOL comparisons between widowed and married women by 5-year age groups

asked about their health more often, were a main caregiver, or were a main source of financial support, they scored higher.

Table 3 presents a second multivariate model that includes interaction terms between marital status and other predictors of QOL including age, sex, education, chronic disease, and family factors. In this model, marital status was not directly associated with PCS or MCS, but several interactions between marital status and other predictors of QOL were significant. For PCS, those who were uneducated, who did not live with their children, and whose children seldom asked about their health suffered more from widowhood. For MCS, those who were male, who did not live with their children, and whose children were a main source of financial support suffered more from widowhood.

\section{Discussion}

\section{Overall QOL scores}

Mean scores for both MCS and PCS in both sexes and all age groups were below the score of 50 that represents the original norm of the scale. The SF12 scale has been shown to have good reliability and validity in China, but it has not 
Table 2 General factorial ANOVA analysis of predictors of PCS and MCS (without interaction terms)

\begin{tabular}{|c|c|c|c|c|c|c|}
\hline & \multicolumn{3}{|l|}{ PCS } & \multicolumn{3}{|l|}{ MCS } \\
\hline & Mean square & $F$ & $P$ value & Mean square & $F$ & $P$ value \\
\hline Sex & 746.68 & 8.54 & 0.004 & 8.27 & 0.12 & 0.729 \\
\hline Age & 3607.45 & 41.25 & $<0.001$ & 205.18 & 2.97 & 0.018 \\
\hline Married versus widowed & 906.38 & 10.36 & 0.001 & 279.48 & 4.01 & 0.037 \\
\hline Education & 597.24 & 6.83 & 0.001 & 873.97 & 12.65 & $<0.001$ \\
\hline Having chronic diseases & $22,225.99$ & 254.14 & $<0.001$ & 5058.64 & 73.24 & $<0.001$ \\
\hline Living with children & 51.76 & 0.59 & 0.442 & 4.32 & 0.06 & 0.803 \\
\hline Children as a main source of financial support & 638.57 & 7.30 & 0.007 & 1043.41 & 15.11 & $<0.001$ \\
\hline Frequency of children asking about their health & 491.82 & 5.62 & 0.004 & 2350.38 & 34.03 & $<0.001$ \\
\hline Children as a main caregiver when ill & 136.90 & 1.57 & 0.211 & 762.06 & 11.03 & 0.001 \\
\hline Overall model significance & \multicolumn{3}{|c|}{ Mean square $=4344.92 ;$ Sig. $<0.001$} & \multicolumn{3}{|c|}{ Mean square $=1302.91 ;$ Sig. $<0.001$} \\
\hline
\end{tabular}

Table 3 General factorial ANOVA analysis of predictors of PCS and MCS (including interaction terms)

\begin{tabular}{|c|c|c|c|c|c|c|}
\hline & \multicolumn{3}{|l|}{ PCS } & \multicolumn{3}{|l|}{ MCS } \\
\hline & Mean square & $F$ & $P$ value & Mean square & $F$ & $P$ value \\
\hline Sex & 231.73 & 2.69 & 0.101 & 0.42 & 0.01 & 0.937 \\
\hline Age & 5202.35 & 60.44 & 0.000 & 253.96 & 3.71 & 0.025 \\
\hline Married versus widowed & 11.17 & 0.13 & 0.719 & 6.80 & 0.10 & 0.753 \\
\hline Education & 942.42 & 10.95 & 0.000 & 579.68 & 8.47 & 0.000 \\
\hline Having chronic diseases & $21,098.13$ & 245.11 & 0.000 & 4110.25 & 60.03 & 0.000 \\
\hline Living with children & 220.73 & 2.56 & 0.109 & 99.07 & 1.45 & 0.229 \\
\hline Children as a main source of financial support & 598.12 & 6.95 & 0.008 & 1308.36 & 19.11 & 0.000 \\
\hline Frequency of children asking about their health & 325.94 & 3.79 & 0.023 & 1787.60 & 26.11 & 0.000 \\
\hline Children as a main care giver when ill & 122.88 & 1.43 & 0.232 & 39.49 & 0.58 & 0.448 \\
\hline Sex $\times$ marriage & 259.71 & 3.02 & 0.082 & 355.51 & 5.19 & 0.023 \\
\hline Age $\times$ marriage & 73.74 & 0.86 & 0.425 & 84.72 & 1.24 & 0.290 \\
\hline Education $\times$ marriage & 332.82 & 3.87 & 0.021 & 11.45 & 0.17 & 0.846 \\
\hline Chronic disease $\times$ marriage & 202.42 & 2.35 & 0.125 & 16.12 & 0.24 & 0.628 \\
\hline Living with children $\times$ marriage & 1389.14 & 16.14 & 0.000 & 429.85 & 6.28 & 0.012 \\
\hline Marriage $\times$ Children as a main source of financial support & 120.64 & 1.40 & 0.237 & 1239.00 & 18.09 & 0.000 \\
\hline Frequency of children caring about health $\times$ marriage & 324.80 & 3.77 & 0.023 & 160.79 & 2.35 & 0.096 \\
\hline Children as main care givers when illness $\times$ marriage & 0.02 & 0.00 & 0.988 & 245.50 & 3.59 & 0.058 \\
\hline Overall model significance & \multicolumn{3}{|c|}{ Mean square $=2849.12 ;$ Sig. $<0.001$} & \multicolumn{3}{|c|}{ Mean square $=914.09 ;$ Sig. $<0.001$} \\
\hline
\end{tabular}

yet been normalized for Chinese populations, let alone specifically for this age group. "Low" mean scores might be due to many factors including age, nuances in wording of translated questions, and/or cultural factors in how respondents answer questions when interviewed. For example, in some cultures elders might gain sympathy by complaining about their health, while in others they might gain respect by being more stoic. Chinese aging populations generally tend to score below international norms on QOL scales [7-9], but this may or may not mean that their level of health-related quality of life is truly lower. Perhaps all that can be said is that there is no indication from these results that overall health-related QOL in these elders is especially high.

\section{Widowhood and QOL}

As hypothesized, QOL was lower in widowed elders than in married elders in most comparisons. This difference was statistically significant in the initial analysis stratified by age and sex for PCS in both men and women and for MCS in men as well as being significant for both PCS and MCS in the combined multivariate model. When looking within age strata, these differences tended to be greater for 
younger elderly widows than for the older elderly, with the exception of PCS for men, which also showed a significant difference in the oldest age group.

MCS was not significantly different overall between widowed and married women. Inspection of Fig. 2 shows a nonsignificant trend toward worse MCS for widowed women for ages $60-69$, which disappears by age 70 . We suspect the overall nonsignificant result may have been due to our study including a substantial number of older widowed women who had been widowed for many years and who had time to adjust psychologically to widowhood in the context of a supportive family environment. This would be consistent with other research suggesting that some of the negative effects of widowhood may decline with time $[26,43]$. MCS in men showed a similar pattern, but differences in age groups under 75 were enough to produce a significant difference for all ages combined. In our study, widowed women tended to be older than widowed men, had probably been widowed for longer, and were more likely to be cared for by their children, perhaps attenuating the impact of widowhood on MCS more in women than in men.

These results are generally consistent with other studies on the effect of widowhood in China and internationally. Chinese studies of widowhood and mortality indicate that men suffer more from widowhood [11-14], as has also been found in England, although studies in North America and Europe did not find differences by gender [16]. This may be due to cultural differences, as previous studies have suggested [31, 32]. In China, women rely on their adult children more than men, who are supposed to be independent, especially economically.

\section{Other predictors of QOL}

Although this was not the main focus of this study, our multivariate analysis identified several other predictors of health-related QOL among these rural Chinese elders, including age, sex, education, and having chronic diseases. These results are consistent with previous international QOL studies among elders [34-38]. Similar to findings in the Chinese middle-aged population [39], family-related characteristics, such as frequency of children's asking about health and whether children are a main source of financial support and care when ill, were also significant predictors of elders' QOL. These results are mostly consistent with a previous study in urban China [11]. One exception is that PCS was higher for elders when children were not a main source of financial support in our study. This may be because elders in better physical health were able to support themselves and did not need financial support from their children.
According to our multivariate analysis, living with children was not by itself a significant predictor of QOL. But it interacted significantly with marital status such that widowed elders had poorer PCS and MCS when they did not live with their children. This is also consistent with previous studies of Chinese elders [12-14]. In general, our interaction analysis suggests that in our sample of elders, who had mostly been widowed for many years, the negative effects of widowhood on quality of life can be mostly mitigated by having a supportive family and to a lesser degree by education.

\section{Limitations of this study}

This study has many limitations. The cross-sectional design can detect associations but cannot identify cause and effect. The measures we used for QOL, while in line with international standards, have not been normalized for elderly Chinese populations, and responses may be biased for the $8.3 \%$ of elders for whom questions were answered by caregivers. Our measures of the family factors that appear to be of great importance are relatively crude. Although our sample was designed to give a broad spectrum of rural environments in China, it does not provide a basis for weighting results to make them representative of rural China overall. Although our sample was fairly large, numbers in age-sex strata were smaller, so that apparent differences by subgroup should be viewed with caution.

\section{Conclusions}

Our results raise concerns for the enormous and rapidly growing population of widowed rural Chinese elders, who have lower physical and mental quality of life than their married counterparts. These elders rely on their children for care, and a supportive family does indeed seem to mitigate negative effects of widowhood on both PCS and MCS. But the rapid increase in the elderly population in rural China coupled with drastically lower fertility rates in recent decades raises questions about whether this model is sustainable. Increasingly, an adult couple may be the only descendants of up to four elders. If this couple migrates to a big city for work or is otherwise unable or unwilling to care for their parents, these elders may have no descendants to care for them at all.

A combination of approaches may be needed to address this situation in the future. Families should be provided with encouragement, resources, and support to help them care for their elders. This might include help from government with the financial burden, visiting nurses to help deal with medical issues and daytime or respite care as is provided for elders in some other countries. In addition, a 
strengthened social safety net must be prepared to care for elders who are no longer able to care for themselves and who do not have family who are willing and able to care for them. This is especially true for widowed elders who may otherwise be left to face the world alone in declining physical and emotional health.

Acknowledgments This research was supported by the Chinese National Social Science Fund (13BRK004).

Authors' contributions JZ was principal investigator of this study and contributed to conception and design, acquisition of data, analysis and interpretation of data, and writing the manuscript. $\mathrm{NH}$ contributed to analysis and interpretation of data and writing the manuscript. All authors read and approved the final manuscript.

\section{Compliance with ethical standards}

Conflict of interest All authors declare that they have no competing interests.

Open Access This article is distributed under the terms of the Creative Commons Attribution 4.0 International License (http://crea tivecommons.org/licenses/by/4.0/), which permits unrestricted use, distribution, and reproduction in any medium, provided you give appropriate credit to the original author(s) and the source, provide a link to the Creative Commons license, and indicate if changes were made.

\section{References}

1. Wang, G., \& Ge, H. (2013). Status of widowed elderly population in China and its development trend. Science Research Aging, 1, 44-55.

2. Zhang, Y. (2013). Family lives, health and care arrangements of Chinese aging population. Jiangsu Social Science, 1, 57-65.

3. Hughes, E., \& Waite, L. (2002). Health in household context: Living arrangements and health in late middle life. Journal of Health and Social Behavior, 3, 1-21.

4. Chappell, N. L. (1991). Living arrangements and sources of caregiving. Journal of Gerontology Social Science, 1, 1-8.

5. WHOQOL Group. (1993). Study protocol for the World Health Organization project to develop a quality of life assessment instrument (WHOQOL). Quality of Life Research, 1993(2), 153-159.

6. Bowling, A., Banister, D., Sutton, S., Evans, O., \& Windsor, J. (2002). A multi-dimensional model of the quality of life in older age. Aging and Mental Health, 4, 355-371.

7. Hao, Z., Chen, C., \& Guo, J. (2014). Quality of Life among community elders. Chinese Journal of Gerontology, 7, 3983-3984

8. Luo, M., Wan, Y., \& Zhang, J. (2011). Health related quality of life of rural and urban elders in Sichuan province, China. Mod Prev Med, 14(2695-7), 2710.

9. Feng, L., Wang, Y., \& Sun, Q. (2014). Multi-factor analysis of quality of life among urban elders in Jilin city, China. Chinese Journal of Gerontology, 9, 4975-4976.

10. Tian, L., \& Zhang, K. (2010). Quality of life and its related factors research among middle-aged and elderly people. Chinese Journal of General Medicine, 13(6A), 1782-1784.

11. Guo, Z., Liu, X., Xu, L., Feng, L., \& Ma, Y. (2015). Quality of life and its relationship with care from children in city. Chinese Journal of Gerontology, 9, 2526-2527.
12. Cheng, X. (2016). Life quality of elderly and living arrangements: empirical study based on CLHLS data. Social Insurance Research, 1, 31-37.

13. Li, J., \& Li, J. (2012). Quality of life research among urban empty-nest elders. J Popul, 3, 31-41.

14. Li, J., Feng, Y., \& Yang, P. (2014). Quality of life research among rural empty-nest elders. Science Research Aging, 5, 3-13.

15. Wei, P. (2010). Subjective life evaluation analysis among elders from different family structures: Based on the survey data of Guiyang city, China. South Population, 5, 32-37.

16. Jiao, K. (2010). The Influence of widowhood on mortality among Chinese elderly. J Popul, 6, 9-13.

17. Huang, Q. (2014). Research on the marriage and death risks of the Chinese elderly. Science Research Aging, 11, 36-43.

18. Gu, D. (2003). Gender difference analysis of marriage impact on health among advanced aged elders. Chinese Population Science, $87,32-40$.

19. Liu, H., Sun, F., \& Hu, P. (1998). National and regional different marital statfus population death characteristic research. Chinese Population Science, 69, 8-15.

20. Li, J. (1994). The marital status and mortality differences of China's population. Chinese Population Science, 44, 16-21.

21. Manzoli, L., Villari, P., Pirone, G. M., \& Boccia, A. (2007). Marital status and mortality in the elderly: A systematic review and meta-analysis. Social Science and Medicine, 64, 77-94.

22. Grundy, E. M., \& Tomassini, C. (2010). Marital history, health and mortality among older men and women in England and Wales. BMC Public Health, 10, 554. doi:10. 1186/1471-2458-10-554.

23. Murphy, M., Grundy, E., \& Kalogirou, S. (2007). The increase in marital status differences in mortality up to the oldest age in seven European countries, 1990-99. Population Studies, 61, 287-298.

24. Stroebe, M., \& Schut Hand Stroebe, W. (2007). Health outcome of bereavement. Lancet, 370, 1960-1973.

25. Onrust, S. A., \& Culipers, P. (2006). Mood and anxiety disorders in widowhood: A systematic review. Aging and Mental Health, $10,327-334$

26. Bennett, K. M. (1997). Widowhood in elderly women: the medium- and long-term effects on mental and physical health. Mortality, 2, 137-148.

27. Thuen, Frode, \& Reime, Marit H. (1997). The effect of widowhood on psychological wellbeing and social support in the oldest groups of the elderly. Journal of Mental Health, 3, 265-274.

28. Byrne, G. J., \& Raphael, B. (1997). The psychological symptoms of conjugal bereavement in elderly men over the first 13 months. International Journal of Geriatric Psychiatry, 2, 241-251.

29. Michael, Y. L., Berkman, L. F., Colditz, G. A., \& Kawachi, I. (2001). Living arrangements, social integration and change in functional health status. American Journal of Epidemiology, 153, 123-131.

30. Pienta, A. M., Hayward, M. D., \& Jenkins, K. R. (2000). Health consequences of marriage for the retirement years. Journal of Family Issues, 21, 559-586.

31. Lev, E. L., \& McCorkle, R. (1998). Loss, grief and bereavement in family members of cancer patients. Seminars in Oncology Nursing, 2, 145-151.

32. Kleinmann, A. (2012). Editorial: culture, bereavement and psychiatry. Lancet, 379, 608-609.

33. Wen, X., Guan, C., \& Liang, J. (2015). Quality of life comparison between empty-nest elders and none-empty-nest elders. Science Research Aging, 2, 1662-1664.

34. Campos, A. C., Ferreirae, E., Vargas, A. M., \& Albala, C. (2014). Aging, gender and quality of life (AGEQOL) study: Factors associated with quality of life in older Brazilian community 
dwelling adults. Health Quality of Life Outcomes, 12, 166. doi:10.1186/s12955-014-0166-4.

35. Sarath, R., \& Sidiah, S. (2015). Quality of life and its determinants among older People living in the rural community in Sri Lanka. Indian Journal of Gerontology, 2, 131-153.

36. Chen, Y., Hicks, A., \& While, A. E. (2014). Quality of life and related factors: questionnaire survey of older people living alone in Mainland China. Quality of Life Research, 5, 1593-1602. doi:10.1007/s11136-013-0587-2.

37. Ozgur, A., \& Aylin, C. (2011). Quality of Life in Ageing Societies: Italy, Portugal, and Turkey. Educational Gerontology, 37, 945-966. doi:10.1080/03601277.2010.492730.

38. Lin, P. C., Yen, M., \& Fetzer, S. J. (2008). Quality of life in elders living alone in Taiwan. Journal of Clinical Nursing, 12, 1610-1617. doi:10.1111/j.1365-2702.2007.02081.

39. Zhou, J., Ru, X., \& Hearst, N. (2014). Individual and householdlevel predictors of health related quality of life among middle- aged people in rural Mid-east China: A cross-sectional study. BMC Public Health, 14, 660. doi:10.1186/1471-2458-14-660.

40. Ware, J. E., Kosinski, M., \& Keller, S. D. (1995). SF-12: How to Score the SF-12 Physical and Mental Health Summary Scales (2nd ed.). Boston, MA: The Health Institute.

41. Lam, E. T., Lam, C. L., Fong, D. Y., \& Huang, W. W. (2013). Is the SF-12 version 2 health survey a valid and equivalent substitute for the SF-36 version 2 health survey for the Chinese? Journal of Evaluation in Clinical Practice, 19(1), 200-208.

42. Xiao, H., \& Kuang, H. (2014). SF - 12 reliability and validity analysis among Chinese elders. Chinese Journal of Gerontology, 2, 1018-1020.

43. Manzoli, L., Villari, P. M., Pirone, G., \& Boccia, A. (2007). Marital status and mortality in the elderly: a systematic review and meta-analysis. Social Science and Medicine, 64(1), 77-94. 